\title{
Automatic Detection of Voltage Notches using Support Vector Machine
}

\author{
Rongzhen $\mathrm{Qi}^{1}$, Olga Zyabkina ${ }^{1}$, Daniel Agudelo Martinez ${ }^{1}$ and Jan Meyer ${ }^{1}$ \\ ${ }^{1}$ Institute of Electrical Power Systems and High Voltage Engineering \\ Technische Universität Dresden \\ 01069, Dresden (Germany) \\ Phone/Fax number: +49 176 31206344, e-mail: rong zhen.qi@mailbox.tu-dresden.de
}

\begin{abstract}
This paper presents a comprehensive framework for voltage notch analysis and an automatic method for notch detection using a nonlinear support vector machine (SVM) classifier. A comprehensive simulation of the notch disturbance has been conducted to generate a diverse database. Based on domain knowledge and properties of power quality disturbances (PQDs), a set of characteristic features is extracted. After feature extraction, a set of most descriptive features has been selected with decision tree (DT) algorithm, and a nonlinear SVM classifier has been trained. Finally, the detection efficiency of the trained model is presented and discussed.
\end{abstract}

Key words. Power quality, feature extraction, voltage notch, decision tree, support vector machine.

\section{Introduction}

Over the past years with the development of modern power systems, an increasing number of power electronic appliances and devices have come into use in both residential and industrial areas. Due to their nonlinear characteristic, these components lead to distortions of the current and voltage, which is one particular phenomenon resulting in a decrease in Power Quality (PQ). The wide variety of $P Q$ phenomena, which cause deviations of voltage, current and frequency in a power grid from the ideally sinusoidal and balanced behavior are defined as power quality disturbances (PQDs). A too strong PQD can result in serious consequences, such as malfunction, damage as well as additional electrical and economic losses. According to a report on PQ in European (EU-25) countries [1], "more than 150 billion Euros" in economic losses are caused annually by PQ problems.

A common PQD, especially in industrial power systems are voltage notches, also called notching. Voltage notch is a steady-state PQD with periodic steep voltage reduction [2]. Voltage notches are caused by the commutation of controlled line-commutating rectifiers and can cause problems in power systems, such as the malfunction of phase sequence relays. Moreover, noticeable oscillations in voltage and current excited by notches (commutation swings) can cause damage to capacitive elements, radio interferences, and malfunctions of precise electronic devices [3]. According to the IEEE 519 standard [4], the two basic indices used to characterize voltage notches are depth and area. A classical notch has a rectangular waveform. However, as presented in previous studies, the waveform may deviate from the classical shape when affected by other network components such as capacitor bank and snubber circuit [3], as shown in Fig. 1.

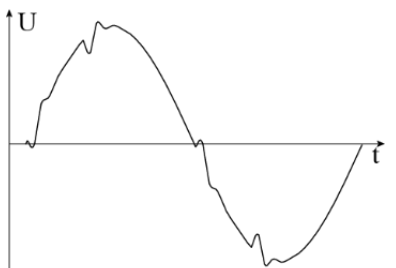

(a)

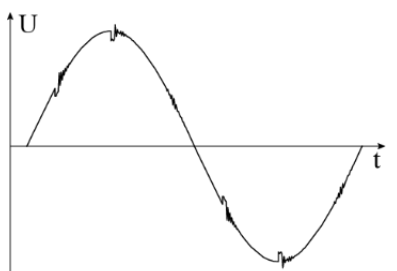

(b)
Fig. 1. Voltage waveforms with non-rectangular notches (a) with capacitive elements; (b) with snubber circuits

In recent years highly automated artificial intelligence (AI) methods have rapidly developed and found wide application in different research fields. AI methods have been applied to automatic detection and classification of different PQDs in PQ measurements and monitoring. The detection of PQDs can be generally divided into two main stages: data representation and classification [5]. In the first stage, the raw data are transformed into an alternative data space with possible dimensionality reduction. For this step, one of the efficient and widely used technique is features-based data representation. With this technique, the raw data are represented by new feature vectors. A wide range of methods to feature-based representations have been developed to facilitate time-series analysis [6] Methods for feature extraction in time series data can be generally divided into three domains: time, frequency and time-frequency domains. Typical time domain methods for feature extraction include stochastic approaches [7], time domain decomposition [8], and piecewise aggregate approximation [9]. Frequency and time-frequency domain methods are based on data transformation, such as Fourier transform, or wavelet transform [10]. Another frequently used transform-based method is space projection, such as phase space reconstruction [11].

Once the time series is transformed into a feature-based representation, a classification stage is performed. Classification presents a supervised task, where a classification model is constructed by learning on a training dataset with available category membership, i.e. labels. The algorithms currently used for PQD 
classification are machine learning methods such as SVMs [12], as well as other AI methods such as fuzzy-logic expert systems (FE-systems) [13] and artificial neural networks (ANNs) [14]. Even though PQD detection using AI method gained a lot of attention, there is a lack of research on notch detection with consideration of various factors influencing the waveform of the notch.

Over the past years very few works were dedicated to analysis and automatic detection of notch phenomena. Therefore, in this work a comprehensive framework for voltage notch analysis and an automatic method for notch detection using a nonlinear support vector machine (SVM) classifier are presented. As result of comprehensive simulation of notch along with other PQDs using MATLAB $^{\circledR}$, a representative waveform database has been constructed. Based on domain knowledge and properties of PQDs, a set of characteristic features is developed. Further, the optimal feature vector was selected with the decision tree (DT) algorithm and used as the input dataset for the SVM classifier.

The remainder of the paper is organized as follows: Section 2 introduces the framework of notch simulation, where synthetic data with notch and other four PQDs are generated. Section 3 gives an overview of the proposed methodology, including feature extraction and selection with DT, and training and validation of the classification model. In Section 4, the developed SVM classifier is applied, and performance results are presented and discussed. Finally, the conclusions are given in Section 5.

\section{Notch simulation}

One of the important aspects of a classification task is availability of a comprehensive waveform database of the studied phenomena. For this work 71 field measurement samples of 10-cycle data have been available, which is not enough to build well generalized classification model. Therefore, to increase the number of available data and build a well generalized classifier, different simulation scenarios of notch have been conducted to supplement the database. Beforehand, theoretical and experimental analysis on influencing factors on the appearance of notches were conducted to have a more comprehensive selection of simulation scenarios for data generation.

As discovered by previous works, there are several parameters or components that influence the appearance of notches, including number of commutations within a cycle, firing angle of commutation, short-circuit power at the connection point, commutation inductance, and loading at the DC side of the rectifier. In addition, the snubber circuits in parallel with the thyristors in the rectifiers and the capacitive elements in the power system such as capacitor banks for reactive power compensation also influence the appearance of notches in the oscillatory frequency and amplitude. The fundamental influencing parameters were theoretically analyzed with a mathematical model as presented in [3]. The two intuitive indices, notch depth $V_{d e p}$ and width $\mu$ are used to describe the shape of voltage notch. The influence of the firing angle $\alpha$, the system and commutation inductance $\mathrm{L}_{\mathrm{S}}$ and $\mathrm{L}_{\mathrm{T}}$, the current at the DC side $\mathrm{i}_{\mathrm{dc}}$, and the amplitude of the voltage source $\widehat{\mathrm{U}}_{\mathrm{m}}$ on the notch depth $\mathrm{V}_{\mathrm{dep}}$ and width $\mu$ has been studied. For example, with $\alpha$ increasing from 0 to $90^{\circ}, V_{\text {dep }}$ increases, while $\mu$ decreases, which means $V_{\text {dep }}$ is positively related to $\alpha$, and $\mu$ is negatively related to $\alpha$. The qualitative summary of effects of influencing factors on the notch depth and area are presented in Table I.

Table I. - Influence of different factors on voltage notch

\begin{tabular}{|c|c|c|}
\hline $\begin{array}{c}\text { Influencing } \\
\text { factor }\end{array}$ & $\boldsymbol{V}_{\boldsymbol{d e p}}$ & $\boldsymbol{\mu}$ \\
\hline$\alpha\left(0\right.$ to $\left.\frac{\pi}{2}\right)$ & Positively related & Negatively related \\
\hline$L_{S}$ & Positively related & Positively related \\
\hline$L_{T}$ & Negatively related & Positively related \\
\hline$i_{d c}$ & Nonlinear & Positively related \\
\hline$\widehat{U}_{m}$ & Positively related & Negatively related \\
\hline
\end{tabular}

In practice, many other parameters and components also significantly influence notching, such as the snubber circuit and the network's capacitive elements. The influence of these components is difficult to analyze with a mathematical model, though possible [3]. Thus, the analysis was supplemented by additional simulations. According to the simulation, the additional capacitors in system have significant effect on eliminating oscillations, while causing global waveform distortion. Moreover, the snubber circuit with lower capacitance and resistance leads to oscillation of higher frequency. In terms of the types of rectifier, the three-pulse midpoint rectifier (M3) has half the notches as six-pulse bridge rectifier (B6) within a period, while the twelve-pulse bridge rectifier (B12) has double number of notches within a period. In particular, the rectifier with uncontrolled full-wave diodes causes much weaker notches than the controlled ones, thus the width and depth cannot be defined clearly.

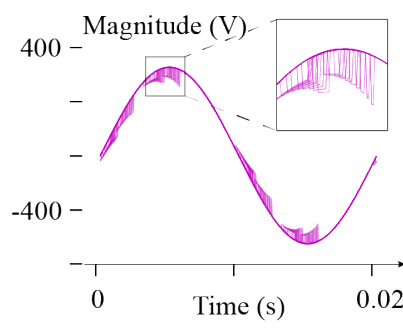

(a)

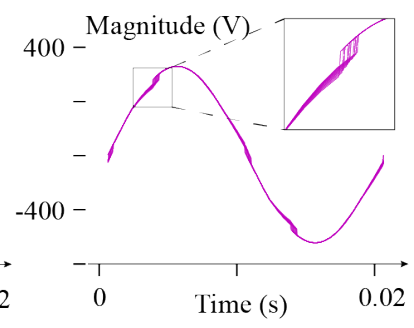

(b)
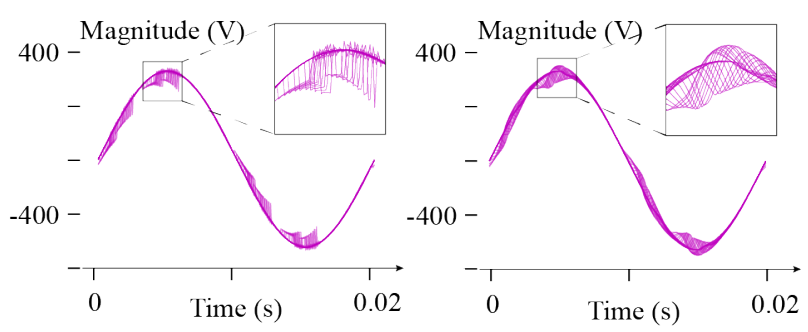

(c)

(d)

Fig. 2. Notch characteristics in synthetic data (a) classical notch; (b) notch with uncontrolled diode; (c) notch with snubber circuit; (d) notch with capacitor. 
Additional waveforms have been generated for the waveform database for three pulse midpoint rectifier (M3), three-phase six-pulse bridge rectifier (B6) and twelve-pulse rectifier (B12). Furthermore, each simulation scenario has four sub-scenarios, corresponding to different waveform characteristics of notches: classical notch, weak notch with uncontrolled diode, notch with waveform distortion caused by capacitors, and notch with oscillations caused by snubber circuit. Overall, 12 scenarios have been simulated, where for each scenario, dozens of samples with different configuration of parameters from Table I were generated. In Fig. 2 examples of overlapped 10 cycles simulated data for B6 and 4 sub-scenarios with varying influencing factors are presented.

To effectively train the classification model, four additional PQDs frequently appearing in power systems, namely sags, swells, harmonics and transients have been simulated. In Table II, the overview on data amounts and PQD are presented. Each sample has a duration of 8 power cycles. Data with notch has been labeled as "notch", while remaining four PQDs as "non-notch".

Table II. - Available input data

\begin{tabular}{|c|c|c|}
\hline $\begin{array}{c}\text { Label of input } \\
\text { data }\end{array}$ & Type of PQDs & $\begin{array}{c}\text { Number of } \\
\text { samples }\end{array}$ \\
\hline \multirow{4}{*}{ Notch } & Notches from M3 & 200 \\
\cline { 2 - 3 } & Notches from B6 & 300 \\
\cline { 2 - 3 } & Notches from B12 & 200 \\
\cline { 2 - 3 } & $\begin{array}{c}\text { Field } \\
\text { measurements }\end{array}$ & 71 \\
\hline \multirow{4}{*}{ Non-notch } & Sags and swells & 240 \\
\cline { 2 - 3 } & Harmonics & 180 \\
\cline { 2 - 3 } & Transients & 120 \\
\hline
\end{tabular}

\section{Methodology}

The proposed methodology includes three steps: feature extraction, model training and validation, and the binary PQD classification with non-linear SVM. The flowchart of the proposed methodology is illustrated in Fig. 3.

\section{A. Feature extraction}

Feature extraction is a process of designing and calculating indices, i.e. features from original or transformed data to capture distinguishing and most characterizing properties of the data, i.e. different PQD. Based on PQ domain knowledge and temporal properties of the data, fourteen features have been developed and calculated. In terms of effective capturing characteristics of the input data, features were derived from original time-series data and momentary derivation time-series data, partly in combination with three other data representations, namely probability density function (PDF), Phase Space Reconstruction (PSR), Fast Fourier Transformation (FFT). All representations and feature calculation have been applied to each sample, i.e., 8 - cycles waveform. The summary of extracted features and prior required transformations is presented in Table III. The momentary deviation time series is defined as:

$$
u_{d e v}(i)=u_{s}(i)-u_{s}^{(1)}(i), \quad i=1,2, \ldots, N_{s}
$$

where $u_{s}(i)$ is the original data, $u_{s}^{(1)}(i)$ is the fundamental wave, $N_{s}$ is the length of one sample and $s$ is sample order.

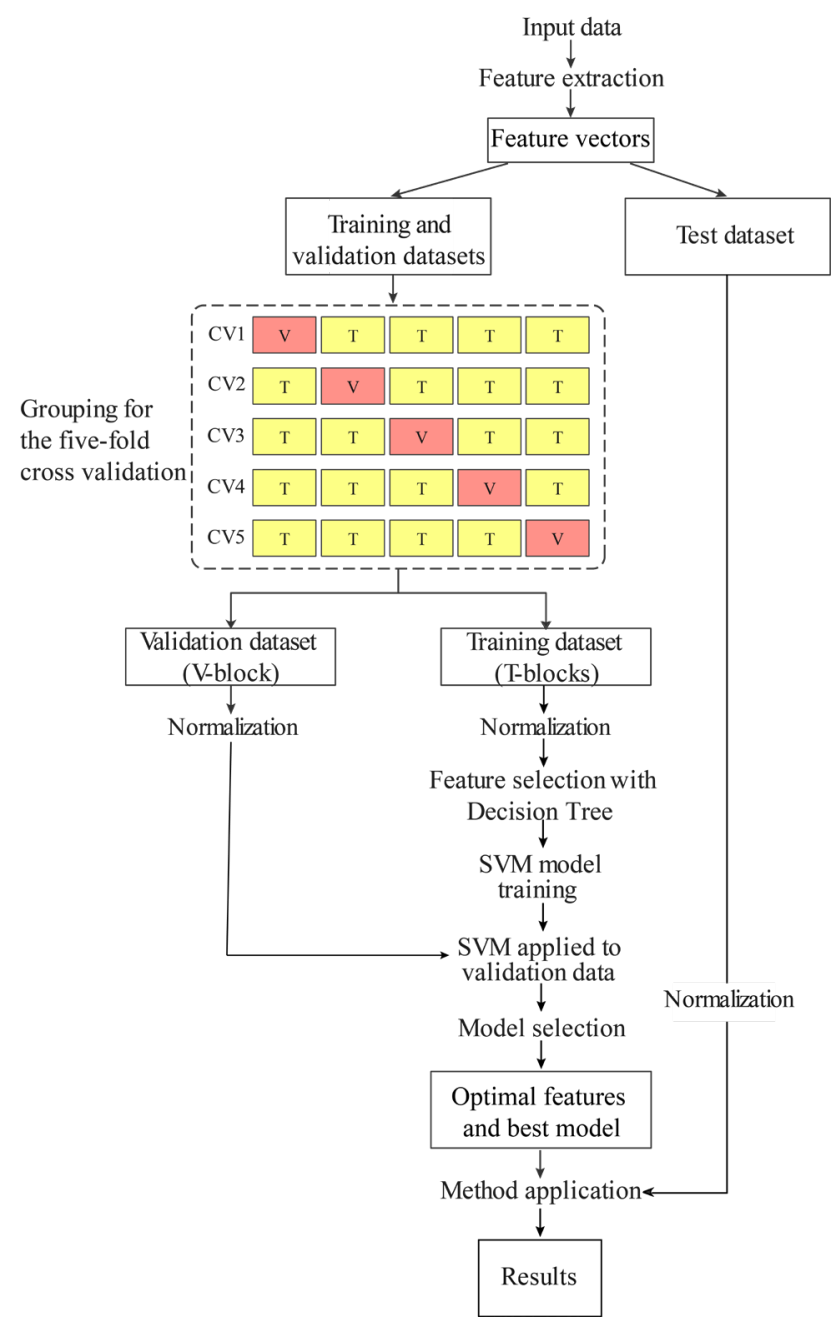

Fig. 3. Process of method development

In order to characterize data properties of different PQDs, first a set of four features namely variance, skewness, kurtosis and Shannon entropy were extracted based on the PDF of the deviation time series. Furthermore, other four features have been extracted already directly from the deviation series such as the waveform index, the energy of the time series, the sample entropy, and the rate of oscillations. The energy feature is proportional to the area between the deviation time series and the time axis. The waveform index is a common parameter used to describe the waveform of a signal, which is calculated as a ratio of the RMS value and the average value of the deviation time series. Sample entropy is a quantity used to estimate the rate at which new information will appear in a time series by calculating the likelihood between subsequences of the time series. It works well with short time series and time series with missing points and can be applied to both stochastic signals and deterministic signals [3]. Finally, the rate of oscillations has been extracted and is defined as the proportion of the number of oscillations in a sample length. Suppose three neighboring points are $u_{d e v}(i-1)$, $u_{d e v}(i)$, and $u_{d e v}(i+1)$ if 


$$
\left[u_{d e v}(i-1)-u_{d e v}(i)\right] \cdot\left[u_{d e v}(i+1)-u_{d e v}(i)\right]>0
$$

then the $i$-th sampled point of the deviation series will be counted as an oscillation point.

Next, from original time series data and in combination with PSR algorithm and FFT a set of six features have been calculated. PSR is a common method used for the analysis of time series data. According to the Tackens embedding theorem, the representation of a dynamic system can be alternatively simplified by projecting a time series of a collection of the states of the dynamic system into a higher dimensional phase space with a proper chosen step length of time delay between each dimension [18]. Based on PSR and a suitable threshold, the feature rate of outranging points has been extracted. It counts the points at the trajectory of the time series, which were located out of a given range and calculated as:

$$
r_{\text {out }}=N_{\text {out }} / N_{s}
$$

where $N_{\text {out }}$ is the number of points falling out of the range:

$$
\left[\widehat{U}_{S}^{(1)}-0.7 \cdot \sigma_{S}, \widehat{U}_{S}^{(1)}+0.7 \cdot \sigma_{S}\right]
$$

where $\widehat{U}_{S}{ }^{(1)}$ is the amplitude of the fundamental wave, $\sigma_{S}$ is the standard deviation of the time series. This rate is closely

\begin{tabular}{|c|c|c|}
\hline $\begin{array}{c}1^{\text {st }} \\
\text { transformation }\end{array}$ & $\begin{array}{c}2^{\text {nd }} \\
\text { transformation }\end{array}$ & Features \\
\hline \multirow{8}{*}{ Deviation series } & \multirow{4}{*}{ PDF } & Variance, $\sigma_{s}^{2}$ \\
\hline & & $\begin{array}{c}\text { Normalized skewness, } \\
S_{S}\end{array}$ \\
\hline & & $\begin{array}{c}\text { Normalized kurtosis, } \\
K_{S}\end{array}$ \\
\hline & & Shannon entropy, $E n t_{s}$ \\
\hline & \multirow{4}{*}{-} & Energy, $\Psi_{\text {devi }}$ \\
\hline & & $\begin{array}{l}\text { Waveform index, } \\
W_{\text {devi }}\end{array}$ \\
\hline & & $\begin{array}{l}\text { Sample entropy, } \\
\text { SampEn }\end{array}$ \\
\hline & & $\begin{array}{c}\text { Rate of } \\
\text { oscillations, } r_{O S C}\end{array}$ \\
\hline \multirow{6}{*}{$\begin{array}{l}\text { Original } \\
\text { data }\end{array}$} & PSR & $\begin{array}{l}\text { Rate of outranging } \\
\text { points, } r_{\text {out }}\end{array}$ \\
\hline & \multirow{3}{*}{ FFT } & $11^{\text {th }}$ harmonic, $\widehat{U}_{S}^{(11)}$ \\
\hline & & $23^{\text {rd }}$ harmonic, $\widehat{U}_{S}^{(23)}$ \\
\hline & & $\begin{array}{l}\text { Total harmonic } \\
\text { distortion, } T H D u\end{array}$ \\
\hline & \multirow{2}{*}{ - } & $\begin{array}{l}\text { Variance of half-cycle } \\
\text { RMS value, } u_{h r, r m s}\end{array}$ \\
\hline & & $\begin{array}{c}\text { Variance of half-cycle } \\
\text { peak value, } u_{p r, r m s}\end{array}$ \\
\hline
\end{tabular}
related to the shape of the time series.

Table III. - Extracted features
As is known from previous experiments [15], the waveform caused by an m-pulse rectifier has characteristic harmonics of the $(m \cdot k \pm 1)$ th order. Hence, $(12 \cdot k \pm 1)$ harmonics are the common harmonics for all three kinds of simulated converters. Therefore, $11^{\text {th }}$ harmonic voltage, $23^{\text {rd }}$ harmonic voltage, and the total harmonic distortion of voltage waveform were extracted from the FFT spectrums of the input data. At last, final two features such as the variance of the half-cycle RMS value and half-cycle peak value were extracted with the consideration of the periodicity of notches.

As result of feature extraction step, 14 features have been developed and calculated over each sample. In total, 1311 samples of 8-cycles data are represented as a matrix of 14 feature time series, where length of each feature time series is equal to 1311 values.

\section{B. Model training and validation}

The model development stage includes model training and validation. To develop and test the classifier, the feature matrix received from feature extraction stage has been divided into two halves. First half of data is used for model training and validation, and second half for model testing. The training and validation have been implemented with a 5 -fold cross validation (CV), aiming at optimizing hyperparameters of the model and avoiding overfitting of the classification. As shown in Fig. 3, each loop of the CV contains a complete training and validation process, including data normalization, feature selection, parameter tuning, and model validation. Data for model training and validation were equally divided into five groups. Within each loop of the $\mathrm{CV}$, one group was used as the validation dataset, and the other four groups together were used as the training dataset. Each feature time series in training and validation datasets has been normalized individually using normalization around mean value:

$$
\widetilde{x_{l}}=\frac{x_{i}-\mu_{T}}{\sigma_{T}}, i=1,2, \ldots k
$$

where $\widetilde{x_{l}}$ is the normalized value for feature $x_{i}, \mu_{T}$ and $\sigma_{T}$ are the mean and standard deviation values of a feature time series $T, k$ is the number of feature values in a feature time series $T$.

Further, the normalized training data was used for parameter tuning of the decision tree (DT), feature selection with the DT, and parameter tuning of the SVM classifier. A DT is a typical supervised optimization algorithm. It divides the dataset into several subsets recursively until some end conditions is met [2]. During the automatic development of a DT, possible splits of the dataset are evaluated with the Gini impurity [16]. This index describes the ability of the features to classify the dataset correctly, which can be considered as a convenient and reliable indicator for the feature selection. The three most important pruning parameters for the end condition of the growth of DT are the maximal depth $d_{\max }$, the minimal number of samples in a leaf node $N_{\text {min, leaf }}$ and the minimal number of samples for further dataset splitting $N_{\text {min,split }}$.

The SVM is one of the most popular supervised techniques for finding the optimal hyperplane with maximal margin to 
Table IV. Results of model validation

\begin{tabular}{|c|c|c|c|c|}
\hline CV No. & $\begin{array}{c}\text { Parameters of DT } \\
\left(d_{\text {max }}, N_{\text {min,leaf }}, N_{\text {min,split }}\right)\end{array}$ & Selected features & $\begin{array}{c}\text { Hyperparameters of } \\
\operatorname{SVM}(C, \sigma)\end{array}$ & $\begin{array}{c}\text { Accuracy for } \\
\text { validation data }\end{array}$ \\
\hline 1 & $(3,1,2)$ & $\widehat{U}_{S}^{(23)}, T H D u, S a m p E n$ & $(18,9.25)$ & $98.54 \%$ \\
\hline 2 & $(4,1,2)$ & $\widehat{U}_{S}^{(23)}$, THDu, SampEn, $W_{\text {devi }}$ & $(17.6,8)$ & $96.35 \%$ \\
\hline 3 & $(4,1,2)$ & $\widehat{U}_{S}^{(11)}, \widehat{U}_{S}^{(23)}$, THDu, SampEn & $(17.8,7.25)$ & $97.81 \%$ \\
\hline 4 & $(4,1,2)$ & $\widehat{U}_{S}^{(23)}, T H D u, S a m p E n$ & $(19.4,9.75)$ & $99.1 \%$ \\
\hline 5 & $(4,1,2)$ & $\widehat{U}_{S}^{(23)}$, THDu, SampEn, Ent ${ }_{S}, r_{o s c}$ & $(19.2,6.5)$ & $94.85 \%$ \\
\hline
\end{tabular}

divide a feature space and is employed in this work. There are linear and nonlinear SVMs, which are suitable for different application scenarios. Due to the possible nonlinear dependencies in the data, the nonlinear SVM with a kernel function was selected to solve the classification problem in this work. With the nonlinear SVM classifier, the classification is conducted in the higher dimensional space, whereas the calculation is implemented in the original lower dimensional space [12]. In this work, the chosen kernel is the radial basis function (RBF), known for its flexibility for high dimensional feature space, defined as follows:

$$
K\left(x_{1}, x_{2}\right)=\exp \left(-\frac{\left\|x_{1}-x_{2}\right\|^{2}}{2 \sigma^{2}}\right)
$$

where $\sigma$ is the width of the Gaussian function. The two hyperparameters of the RBF-SVM that must be tuned are the regularization parameter $C$ and $\sigma$. The grid search algorithm was used for parameter tuning of both the DT and SVM.

Once the classifier is trained within each loop of $\mathrm{CV}$, it is further applied to the validation dataset to evaluate the performance of the classifier. As an outcome of each validation stage, the parameters of DT, selected features and hyperparameters of SVM have been received and listed in the Table IV. As it can be seen from the table, parameters of DT and SVM stay relatively stable as well as a set of selected features, i.e. features such as $\widehat{U}_{S}^{(23)}, T H D u$ and SampEn. High classification performance of the developed method is observed in all 5 cases of validation, i.e. more than $96 \%$. However, the highest performance has been reached with model in $\mathrm{CV} 4$, i.e. $99.1 \%$, which has been selected for final method application on testing data set.

\section{Method Application and Results Analysis}

According to validation results in Section 3.B the features and SVM model of CV 4 are used. Once feature time series is normalized using the mean value and standard deviation from the training data, SVM classifier has been applied to the normalized dataset. It is important to mention, as nonlinear SVM presents a binary classifier two types of labels have been assigned to the data. Data with notch distortion is labeled as "notch" and data with sags, swells, harmonics and transients are labeled as "non-notch".

The total and per class accuracy are calculated and presented in the Table V. As it can be seen from the table, the developed SVM classifier has high total accuracy of $99.2 \%$, and per class accuracy of $99.24 \%$ and $99.14 \%$, respectively. Only a slight difference in accuracy for each class can be noticed. For notch data class, the decrease is equal to $0.8 \%$ with 3 misclassified samples as non-notch. Whereas for non-notch class, the misclassification is equal to $0.9 \%$, i.e. 2 samples out of 234 are misclassified as notch. Due to the lack of real data, only 19 samples from real measurement data with notch remained in the test dataset, but all of them were correctly classified. Consequently, all 5 misclassified samples belong to the synthetic dataset.

Table V. Results of model application to test data

\begin{tabular}{|c|c|c|c|}
\hline Class & Notch & Non-notch & Both \\
\hline Accuracy & $\frac{391}{394}=99.24 \%$ & $\frac{232}{234}=99.14 \%$ & $\frac{623}{628}=99.20 \%$ \\
\hline
\end{tabular}

Three misclassified non-notch samples were swells originated from the energizing of a capacitor bank in the network. The possible reason of the misclassification can be an accompanying impulsive transient during the switching operation of capacitor banks upon closing of breakers, whereas two misclassified notches were simulated with $\mathrm{M} 3 \mathrm{C}$ converters. The closer look on the data has revealed that the depth of the misclassified notches is slightly lower compared to correctly classified notch.

In the Fig. 4 the values of three calculated features over testing data is presented as $3 \mathrm{D}$ plot. The correctly classified notch and non-notch samples are depicted as blue and green circles, while misclassified notch and non-notch samples with blue cross and red triangle, respectively. As it can be seen from the Fig.4, the feature values for all 5 misclassified cases are located in the overlapping area of notch and non-notch data.

The general distribution of the feature values can be interpreted with the theoretical basis behind each feature. Exemplarily, the sample entropy is a quantity used to describe the chaos of a dynamic system, i.e. a sample with higher structural complexity is expected to have a higher sample entropy. Such higher values of sample entropy can be observed in data with harmonics compare to the data with sag, swell and transient PQDs. Sags and swells tend to have the lowest sample entropy and narrow spectrum due to their almost sinusoidal waveform. Exceptionally, the phase angle jump (PAJ) of the voltage occurs with sags when the ratio of system resistance and inductance changes after switching off, inserting large loads, or three-phase fault [17], which can be described as an abrupt voltage loss and phase shift. In this case, sag and swell samples have a 
more widely and evenly distributed spectrum than other non-notch data. However, it is important to mention that due to nonlinear feature projection of the RBF-SVM, result interpretation becomes challenging and is not always easily possible.

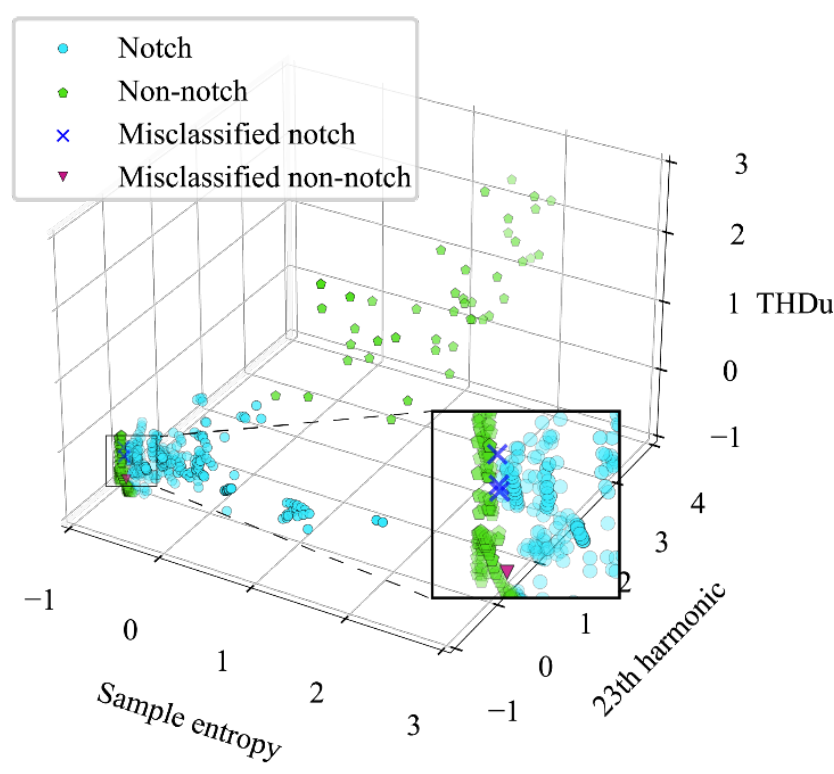

Fig. 4. Visualization of the feature space

\section{Conclusion}

This paper proposes a comprehensive framework for voltage notch analysis and automatic detection based on nonlinear support vector machine classifier. A comprehensive simulation framework for notching phenomena resulted in deeper understanding of factors influencing notch depth and area, which are the most common parameters to characterize notches. Additionally, as result of simulation, it was possible to increase waveform data diversity build a well generalized classification model. According to results of the method performance, the feature extraction based on both domain knowledge and time-series properties provides several advantages such as deeper understanding the data properties of PQDs, reduction the data size and computation time over the conventional approaches. In addition, an efficient feature selection algorithm such as decision tree can highly reduce the feature vectors, decreasing the risk of overfitting and saving considerable memory space and computational time. Furthermore, the constructed classifier is able to classify notch and non-notch waveforms with high accuracy of $99.2 \%$.

In future work, several potential improvements can be done for the notch detection and classification. For example, localization of notching source and application of a multiclass support vector machine to a more diverse database of power quality disturbances.

\section{References}

[1] O. P. Mahela, U. K. Sharma and T. Manglani, "Recognition of Power Quality Disturbances Using Discrete Wavelet
Transform and Fuzzy C-means Clustering," 2018 IEEE 8th Power India International Conference (PIICON), Kurukshetra, India, 2018, pp. 1-6.

[2] IEEE Recommended Practice for Monitoring Electric Power Quality, in IEEE Std 1159-2009, vol., no., pp.1-94, 26 June 2009.

[3] R. Ghandehari and A. Shoulaie, "Evaluating Voltage Notch Problems Arising from AC/DC Converter Operation," in IEEE Transactions on Power Electronics, vol. 24, no. 9, pp. 2111-2119, Sept. 2009.

[4] IEEE Recommended Practice and Requirements for Harmonic Control in Electric Power Systems, in IEEE Std 519-2014, vol., no., pp.1-29, 11 June 2014.

[5] S. Khokhar, A. A. M. Zin, A. S. Mokhtar, N. A. M. Ismai and N. Zareen, "Automatic classification of power quality disturbances: A review," 2013 IEEE Student Conference on Research and Developement, Putrajaya, 2013, pp. 427-432.

[6] F. Ben, "Feature-based time-series analysis," Oct. 2007.

[7] Manimala, K., Selvi, K., \& Ahila, R. (2012), "Optimization techniques for improving power quality data mining using wavelet packet-based support vector machine." Neurocomputing, 77, 36-47

[8] T. Yalcin, O. Ozgonenel and U. Kurt, "Feature vector extraction by using empirical mode decomposition for power quality disturbances," 2011 10th International Conference on Environment and Electrical Engineering, Rome, 2011, pp. 1-4.

[9] E. Styvaktakis, M. H. J. Bollen and I. Y. H. Gu, "Automatic classification of power system events using RMS voltage measurements," IEEE Power Engineering Society Summer Meeting, Chicago, IL, USA, 2002, pp. 824-829 vol.2.

[10] K. Suhail, M. Z. Abdullah, M. Ahmed and Zareen N. "Automatic Pattern Recognition of Single and Multiple Power Quality Disturbances," Australian Journal of Electrical and Electronics Engineering, 2015.

[11] M. Kolenc, E. Plesnik, J. F. Tasič and M. Zajc, "Voltage notch detection and localization in power quality signals in phase space," Eurocon 2013, Zagreb, 2013, pp. 1745-1752.

[12] P. G. V. Axelberg, I. Y. Gu and M. H. J. Bollen, "Support Vector Machine for Classification of Voltage Disturbances," in IEEE Transactions on Power Delivery, vol. 22, no. 3, pp. 1297-1303, July 2007.

[13] M. G. Amouzad and Mohamed Azah, "A Fuzzy-Expert System for Classification of Short Duration Voltage Disturbances," Jurnal Teknologi, 2012.

[14] S. Mishra, C. N. Bhende and B. K. Panigrahi, "Detection and Classification of Power Quality Disturbances Using STransform and Probabilistic Neural Network," in IEEE Transactions on Power Delivery, vol. 23, no. 1, pp. 280-287, Jan. 2008.

[15] D. D. Shipp and W. S. Vilcheck, "Power quality and line considerations for variable speed AC drives," in IEEE Transactions on Industry Applications, vol. 32, no. 2, pp 403-410, March-April 1996.

[16] Strobl. C. Boulesteix, A.-L., and Augustin. T., "Unbiased split selection for classification trees based on the Gini impurity," Computational Statistics \& Data Analysis, 52(1), 483-501, 2012.

[17] Y. Wang, M. H. J. Bollen and X. Xiao, "Calculation of the Phase-Angle-Jump for Voltage Dips in Three-Phase Systems," in IEEE Transactions on Power Delivery, vol. 30 , no. 1, pp. 480-487, Feb. 2015.

[18] Takens, F., "Detection strange attractors in turbulence," Lect. Notes Math., 1981, 898, pp. 366-381 